\title{
Advances and trends for the development of ambient-assisted living platforms
}

\author{
Angelo Costa, ${ }^{1 *}$ Vicente Julián ${ }^{2}$ and Paulo Novais ${ }^{2}$ \\ (1) Centro ALGORITMI/Departamento de Informática, Escola de Engenharia, Universidade do Minho, Portugal \\ E-mail: acosta@di.uminho.pt \\ (2) Departamento de Sistemas Informáticos y Computación, Universitat Politècnica de València, Camino de Vera \\ s/n46022 Valencia, Spain
}

\begin{abstract}
Ambient Assisted Living (AAL) and Ambient Intelligence (AmI) try to achieve a future where technology surrounds the users and helps them in their daily lives. In this sense, the urgent need of solutions to cover the rapid increase of the elderly population with chronic diseases led to the increase of projects related with AAL and AmI. During the latest years, several projects have been proposed to tackle different medical problems, some building devices and others services. This paper presents iGenda and its evolution, the UserAccess, with the main objective of developing an AAL platform. It features an analysis of the latest developments and points future directions for the work. These projects display the importance of the interoperability of the platforms, demonstrating a case study for AAL development.
\end{abstract}

Keywords: Ambient Assisted Living, Ambient Intelligence, Intelligent Envi-ronments, AAL4ALL, e-Health, Active Ageing, Artificial Intelligence

\section{Introduction}

The European Union (EU) defined the year of 2012 as the year of active ageing, ${ }^{1}$ where social efforts and funding were directed to solutions that aimed to solve different agerelated problems. This urgent need was uncovered by recent United Nations reports about population evolution (United Nations, 2009; United Nations, Department of Economic and Social Affairs, P.D., 2015; Department of Economic and Social Affairs, P.D., 2013). These reports showed that the elderly population is rapidly increasing and the younger population (as in pre-teenagers) is rapidly decreasing, and showing that the population over 60 years old will double by 2050 as Figure 1 shows. Another ageing problem is the declining fertility.

The human being longevity is determined by several factors, like the quality of medical care, economic stability and social care. The question that remains is what is the most important factor to this increase in longevity?

The Department of Economic and Social Affairs, PD (2013) shows that in developing countries, the elderly population numbers are increasing even if they lack the medical, social and economic resources.

The only factor that is commonly shared between the countries on the United Nations report is the decrease of the fertility values. Thus, as seen in Figure 2, the population

\footnotetext{
${ }^{1}$ http://ec.europa.eu/archives/ey2012/
}

distribution becomes slimmer at the bottom (in the visual form of a pyramid) and balanced at the middle and top.

The panel for Health Ageing and Retirement in Europe conducted a survey that stated that at least two chronic diseases were found on $40 \%$ of people aged over 50 years (Adena \& Myck, 2014; Mair et al., 2015; Boffetta et al., 2014), evidencing that it is much cheaper to invest in prevention and proactivity, acting as soon as possible to keep the population from having serious medical conditions.

To overcome some situations where help or assistance was scarce, some home environments were equipped with sensor systems to respond quickly to critical situations, such as falls where one may not be able to call for help.

The aim of this paper is to present two projects, the iGenda and the UserAccess (UA), and make a parallel between the current ambient-assisted living (AAL) developments with the aim of foreseeing the roadmap of this kind of systems. We also present the Care4Balance, understAID and RelaxedCare projects, which are very similar to the iGenda or the UA, in terms of architecture or concept. The analysis of these platforms highlights the positive and negative features of each one, their similarities and how they can be an advantage for the future developments.

The paper is structured as follows: Section 2 introduces the concepts of Ambient Intelligence (AmI) and AAL and an analysis of the selected projects; Section 3 presents the iGenda and Section 4 the UA. Finally, Section 5 shows the conclusions and future paths. 


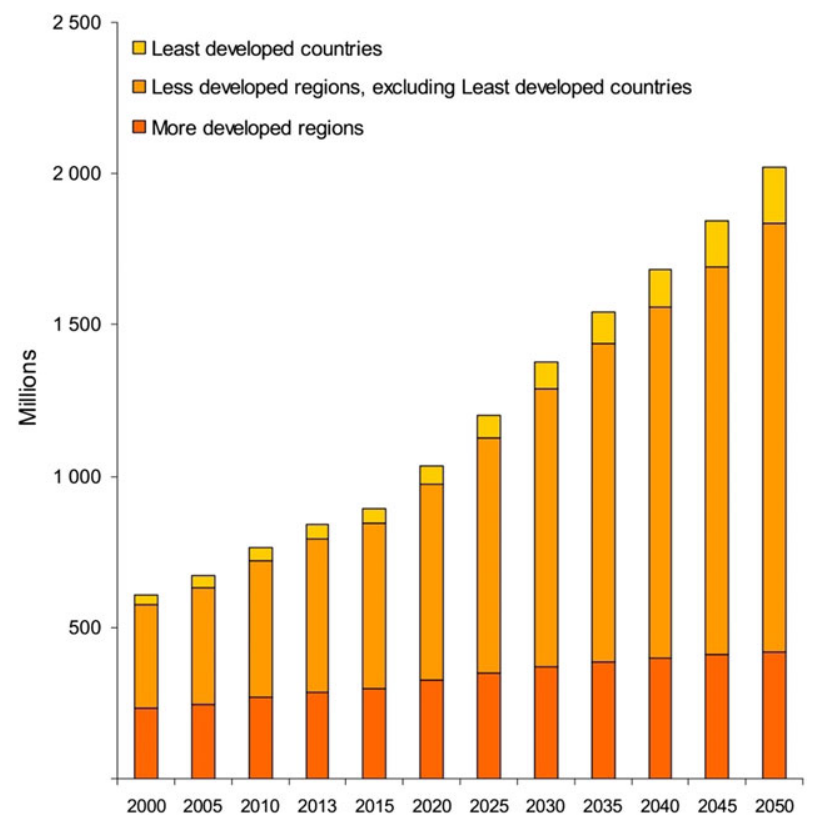

Figure 1: Distribution of population aged 60 or over by regions, 2000-2050 (Department of Economic and Social Affairs, P.D., 2013).

\section{Related work}

This section presents the related work. First is the AmI and the AAL areas. They show the social impact these projects have. Second is the most relevant related projects that represent other efforts that are currently being made. The differences of their concepts and architectures in relation to iGenda and UA are identified and exposed.

\subsection{Ambient Intelligence and Ambient Assisted Living}

Ambient intelligence responds to the technological call for monitoring and acting on the home of people with disabilities. Its aim is to create a nest of sensor systems that together could provide contextual information, thus transforming data into knowledge (Aarts \& Encarnação, 2006; Aarts et al., 2001; Cook et al., 2009). This is achieved by creating digital environments that are sensitive to people's needs and can respond to their requirements, anticipate behaviours and adjust the response accordingly.

In the last years, several projects have been developed to attend to the needs of AmI. The advances of the Internet of things have opened new hypotheses of integrating home appliances in home networks where they can be remotely controlled (Kranz et al., 2010; Gershenfeld et al., 2004; Atzori et al., 2010).

Ambient Assisted Living has spawned as a subset of AmI with the goal of creating proactive environments for the elderly and people with disabilities. This separation occurred to convey the attention to the limitations and requirements of these people, mainly concentrating the efforts on personalization. AAL differs from AmI because AmI requires that physical environments adapt to every person and AAL requires that physical environments adapt
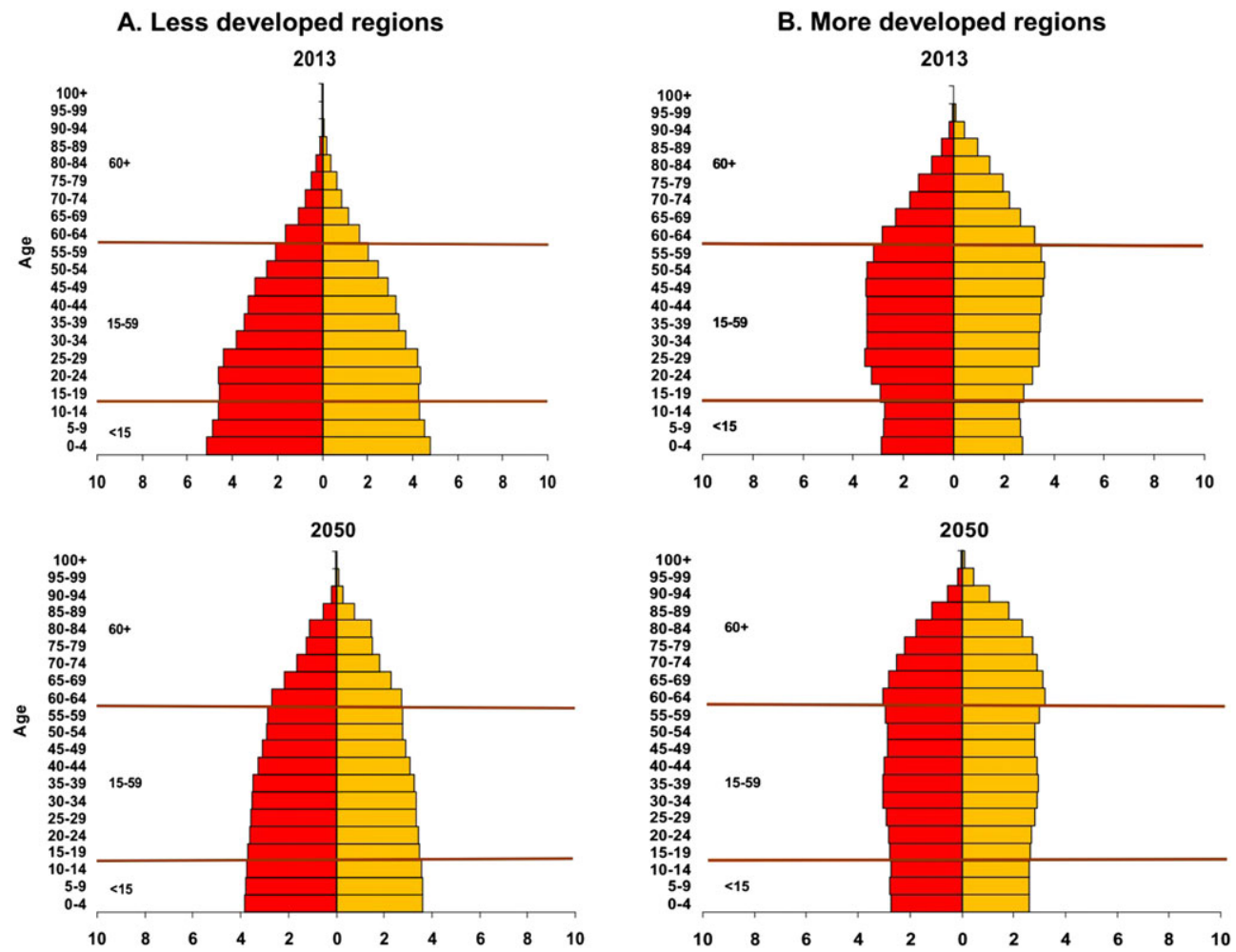

Figure 2: Population pyramids of the less and more developed regions of 2013 and 2050 (Department of Economic and Social Affairs, P.D., 2013). 
to a specific person (Vastenburg et al., 2008; Ramos et al., 2008; Cook et al., 2009).

One of the great investors in this idea was the EU, distributing several funds to the research of AAL projects. For instance, one of the funded is the Ambient Assisted Living Joint Programme, which is a consortium of countries that promote and invest in AAL projects. These actions promote the novelty factor and compel the flourishing of ideas that later may be translated into devices or services.

\subsection{Related projects}

In the multitude of AAL projects, there are some important projects that need to be referred in order to show the different existing perspectives. AAL is a broad area of application, which means that even though most of the projects are related, they tend to differ in the aim, architecture or concept. The following projects show different facets of AAL developments and follow closely the iGenda and UA concept.

2.2.1. Care4Balance The Care4Balance (Care4Balance, 2015) project consists in an aggregator of information directed to the care receivers and the formal or informal caregiver. The goal is to connect every user with their circle of trust, sharing information about everyday events with the additional feature of detecting problematic situations that may occur.

The architecture is defined in a client-server style, where the server maintains all information and ensures the data flow between each client. The Care4Balance client is expected to be operating in a mobile device, but the authors are not very clear about its features or if it will be the only method of access. This project is still active and is now in the final stages of field trial. The main issue with this project is the way it approaches the users in terms of visual interfaces. The coloured cube is not very informative and may be forgotten by people with cognitive disabilities. In search of minimal interaction, they have minimized too much. Additionally, they lack interoperability features.

2.2.2. UnderstAID The understAID (UnderstAid, 2015) is an EU-funded project that focuses on the development of an e-leaning application that provides information about medical conditions directed to the dementia disease. The authors claim that there is an absence of an effective search method about medical information and that the information that exists is too technical to non-medical people.

This project is designed for informal caregivers by providing them with a comprehensive library of procedures and general information about degenerative diseases. The personalization is performed through surveys and profiling the care receiver, generating a list of correct procedures to each user. This project is currently at its final stage, having a beta application publicly available for testing. This project is only directed to the caregivers, which leaves the care receivers without any help. Furthermore, the level of interaction is very limited.

2.2.3. RelaxedCare The RelaxedCare (RelaxedCare, 2015) project has as a goal to establish a social connection between the elderly and its relatives and create a strong social bond. To achieve this goal, it uses the architecture of the HOMER project (Fuxreiter et al., 2010; Fuxreiter et al., 2011; HOMER, 2015) to convey the users' information benefitting from their concept of 'AAL in a box' (off-the-shelf products that can be bought directly from any vendor).

The platform receives messages from the relatives and notifies the care receivers through actuators, noticeably, performed through a coloured cube. Furthermore, the caregivers can access a sensor platform to view the current status of the care receivers, now represented by a tracker for lost objects, such as keys or glasses. The latest development news is that the project is being tested in a controlled environment. This project is limited in terms of real help. The social network provides inclusion and social cohesion, but it is unclear that it has an impact on the users' lives.

\section{3. iGenda}

The iGenda (Costa \& Novais, 2011; Costa et al., 2011; Costa et al., 2010a) has as main goal to provide intelligent event management. It consists in a platform that receives events from its users and tries to schedule them according to their importance, having the ability of creating, moving and deleting events. Moreover, it supports shared events and can schedule ludic activities in the users' free time adjusted to their medical condition. This last feature was developed to promote active ageing.

The iGenda spawned from the VirtualECare project (Costa et al., 2010b), with the initial intention of generating a visual interface to the platform services. The verified performance and the good reception from the scientific community made clear that the iGenda should be an independent project. For instance, it was able to receive information and process it according the user profile and the event importance.

The following scenario was possible: upon receiving a high priority event from a physician, the system reallocated any conflicting event to other time slot, and the high-priority event was scheduled in the specified time.

\subsection{Architecture}

In terms of architecture, the iGenda followed a typical client-server structure, where the client acts as the visual gateway to the platform, leaving all the heavy processes to the server. The client was designed initially as a desktop application, due to the low proliferation of mobile devices at the beginning of the project. The last update of the client 
introduced a web interface, a modular endpoint to both desktop and mobile platforms.

The client has two main features: visualizing the events as a list of activities and the creation of shared events with other users. The platform worked with the known ISO ical (RFC 5545 and updates RFC 5546 and RFC 6868) facilitating the use of external applications for visualization of such files. This decision was made, so it reduces the users' interactions, keeping the act of using the iGenda simple, which means increasing the possibilities of its success. Figure 3 shows an overview of the iGenda architecture.

The server is constituted by three major modules: the agenda manager (AM), the conflicts manager (CM) and the free time manager (FTM). These modules and the platform in general are constituted by multi-agent systems (MAS) (Camarinha-Matos \& Afsarmanesh, 2004; Wooldridge, 1997) established in Java Agent DEvelopment Framework (JADE) (Gawinecki \& Frackowiak, 2008; Bellifemine et al., 2007). The MAS allow the creation of independent modules and plug-and-play features, each one bounded to their own agent. MAS make deployment and maintenance easier as they are independent and reliable, bypassing any agent that is causing errors and rebooting it. Furthermore, high-level agents can be used to monitor the agents, automating maintenance tasks in case of errors.

The architecture is hierarchical; there is only one AM per server (an array of servers is recommended for load balancing) and a pair of CM and FTM for each user. This is a platform with agents-inside-agents, in which each agent contains other internal agents that process the information according to their role. This type of architecture acts using strict ontologies, exchanging human-readable messages when possible.

In terms of the modules, their tasks are as follows:

- AM: it is in charge of receiving the information of each client (typically a new event) and relaying it to the appropriate $\mathrm{CM}$ module. It also responds to each client in case of important actions occurred (such as critical remainders or removal of an event). Furthermore, it is responsible for verifying the integrity of all $\mathrm{CM}$ and FTM available and stopping and restarting them if needed.

- $\mathrm{CM}$ : it is divided in three processes, fetching, reallocating and saving. The fetching consists in allocating the user calendar and search for the week that the event is referring to, locating the events that collide with it. The reallocating calculates a balanced priority with the importance value of the event and the user importance. The most important events stay static, and the least important ones are moved to other timeframe or, in extreme cases, deleted. Lastly, the saving processes the changes and uploads them to the user calendar and notifies the AM, and the AM too notifies the user(s) if that is the case.

- FTM: this module operates silently, and its task is to schedule playful events on the user's calendar to promote an active life. It fetches the events on the user-defined database that has activities that the user likes or requires and schedules activities randomly but being sensible to the daytime hours (this avoids that certain tasks, like going outside, occur during the night-time).

The modularity and operation as a service allow the use of the iGenda as a base platform to other systems. For instance, a localization platform (Ramos et al., 2012; Ramos et al., 2014) uses the information of the events and the client's interface to deliver information to the user. The iGenda project has led to different advances, namely, supporting systems that take advantage of its features, and a direct evolution of the iGenda: the UA.

The iGenda project served as the base structure for the UA project. The UA project is different in terms of its aim, but the core architecture relies on the iGenda. The UA is explained with more detail in the next section.

\section{UserAccess}

The aim of UA is to provide intelligible information directed to the caregivers about the care receiver (Costa et al., 2014). The UA is a natural evolution of the iGenda, taking advantage of its architecture and communication methods.

The UA was developed for the AAL4ALL project (AAL4ALL, 2015), a Portuguese consortium of private and public institutions, with the goal to provide easy-touse, plug-and-play AAL solutions (either software or hardware) directed to the elderly community. Some of the developments were house-sensor platforms, body sensors and transparent services that collect, process and show information.

The main issue while working with elderly is that they have several limitations, requiring a social conduct designed especially for them, as most of the elderly population are not prone to work with advanced technological devices.

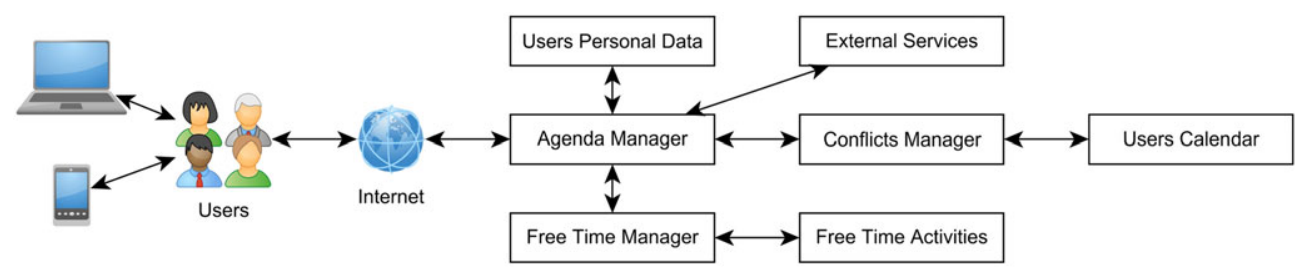

Figure 3: iGenda architecture overview. 
Furthermore, there are social aspects that have to be specially considered, like the health concerns that must be first announced to the physician or the caregivers before (if at all) it is showed to the care receiver. The field tests performed for the AAL4ALL suggested that some information would be more effective if it were delivered and explained by a person that the elderly trusts rather than by an application.

One of the issues is that the available information services directed at the caregivers are cumbersome and difficult to use, being most of them only internally available and lacking remote access. This results in low acceptance by the users, which only use the service when forced, for example, a scenario that is common in nursing homes mostly is to keep track of the patients' personal health record.

The UA works under the consideration that it is required that the caregivers maintain their assigned care receiver under constant monitoring and have a limit to the number of monitored people.

The lack of support for caregivers sets the tone for the development of the UA, which was designed to show information that is understandable, easy to read and is adjusted to each user. For instance, the information delivered to a formal caregiver can be medical oriented and include sensitive information; an informal caregiver may only receive overall notifications about the care receiver status and warnings from the home sensors (if a window is open and the user is not at home). Moreover, the care receivers can use this application to verify if there are any warnings or to check their personal health record.

The UA accepts heterogeneous communication through the use of marked-up message content. It has predefined ontologies and can receive communication from external services that wish to use the inference engine or the visual interfaces. It has also the ability to schedule events using the iGenda platform.

\subsection{Architecture}

The UA focuses on the interfaces, and it was developed natively for the Android platform and for the web. The webpage has more information and serves as the administration platform. The user can access the administration page to enable/disable sensor platforms and personalize the warnings messages. The mobile version is designed to be simple and to present a condensed version of the information, concentrating in the warnings. Figure 4 shows the Android application, with the main window of information and the widget, which is a quick view of the current warnings. Figure 5 shows the administration page of the caregiver view where the information is displayed and can be configured. Also, both visual interfaces can manage the information of several users, allowing one platform to provide information of several users.

The architecture follows a client-server structure and uses MAS on the server. The MAS serve not only for security purposes but also to easily deploy new features. For instance, if a new sensor platform is added to the environment, like cameras, a data parser was provided to the UA to interpret the input and generate the UA compatible output. The MAS enforce the idea of distributed computing, allowing the distribution of each agent in a different computer.

Following the base requirements of the AAL4ALL, the UA structure overview is showed in Figure 6. In the carereceiver block, the body sensors and home sensors are directly connected to the AAL4ALL, apart from being required by the project. This ensures the interoperability of the system. Any vendor has to assure that their products operate according to the AAL4ALL specifications. The AAL4ALL enforces certification to integrate a new device; thus, each device has to have the appropriate data nodes, so any other service can access to that information. The UI device connects directly to the RabbitMQ (RabbitMQ,

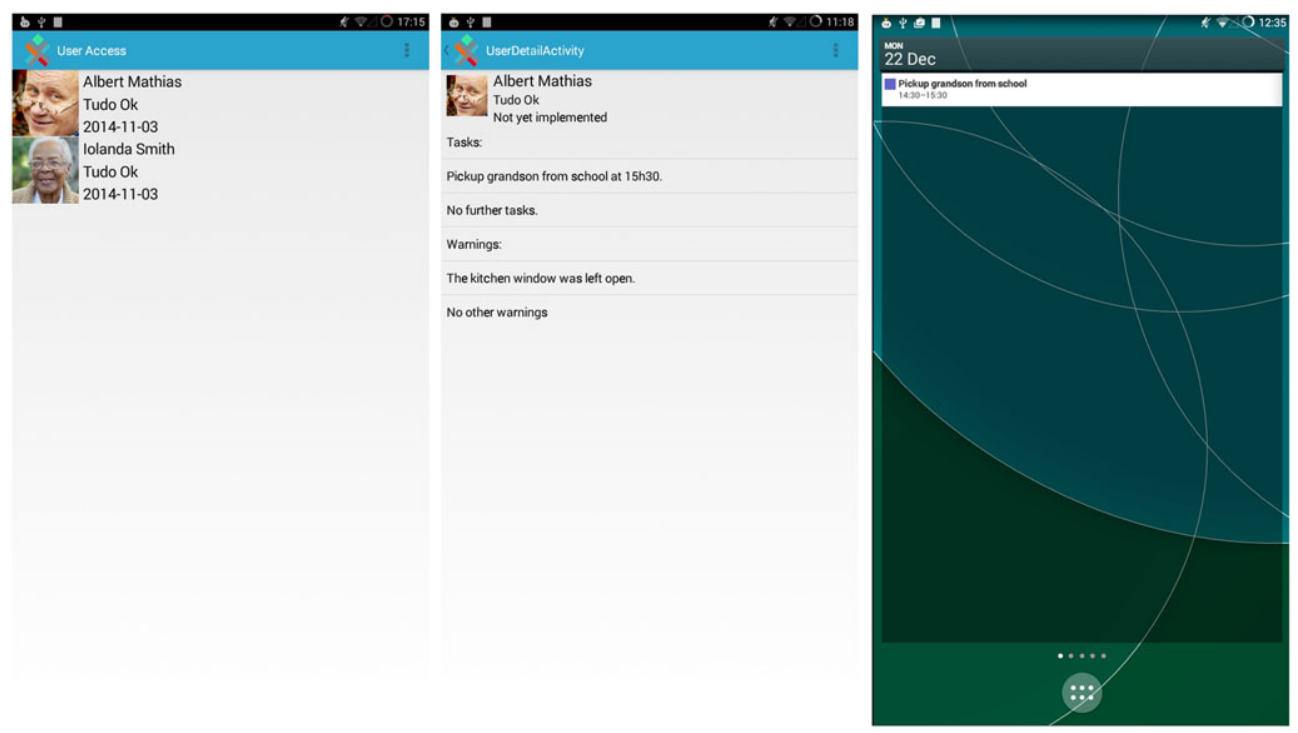

Figure 4: UserAccess mobile interfaces. Left: user selection; middle: selected user warnings and personal details; and right: warnings widget. 


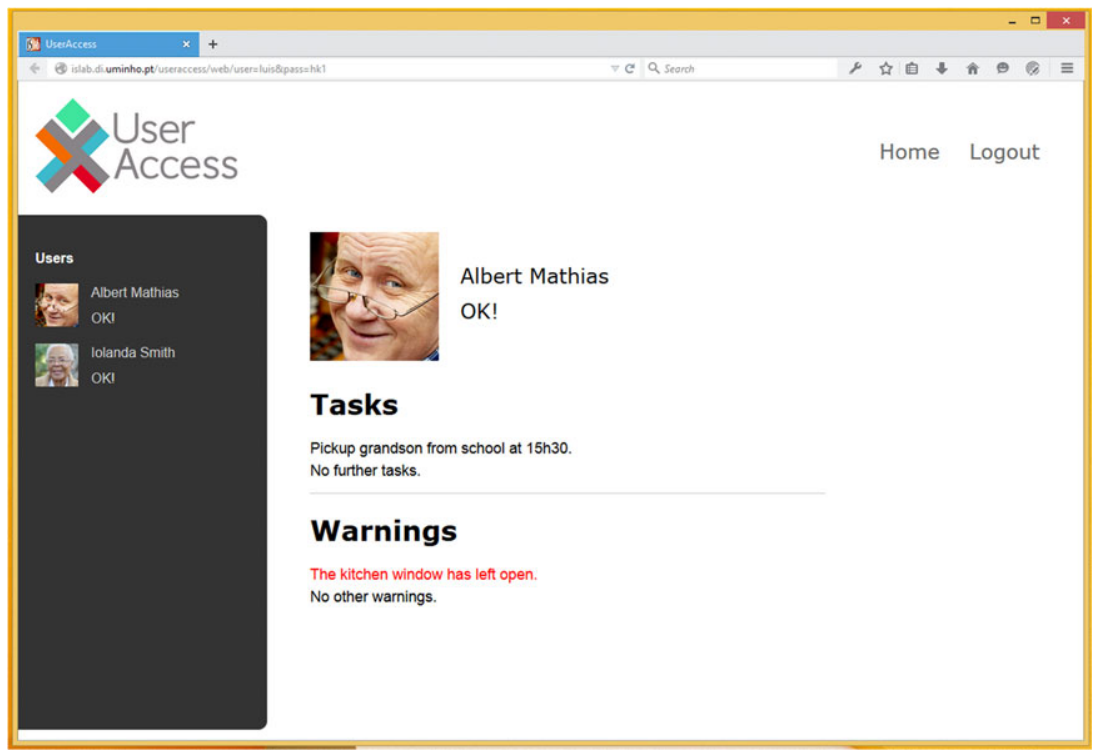

Figure 5: UserAccess administration website.

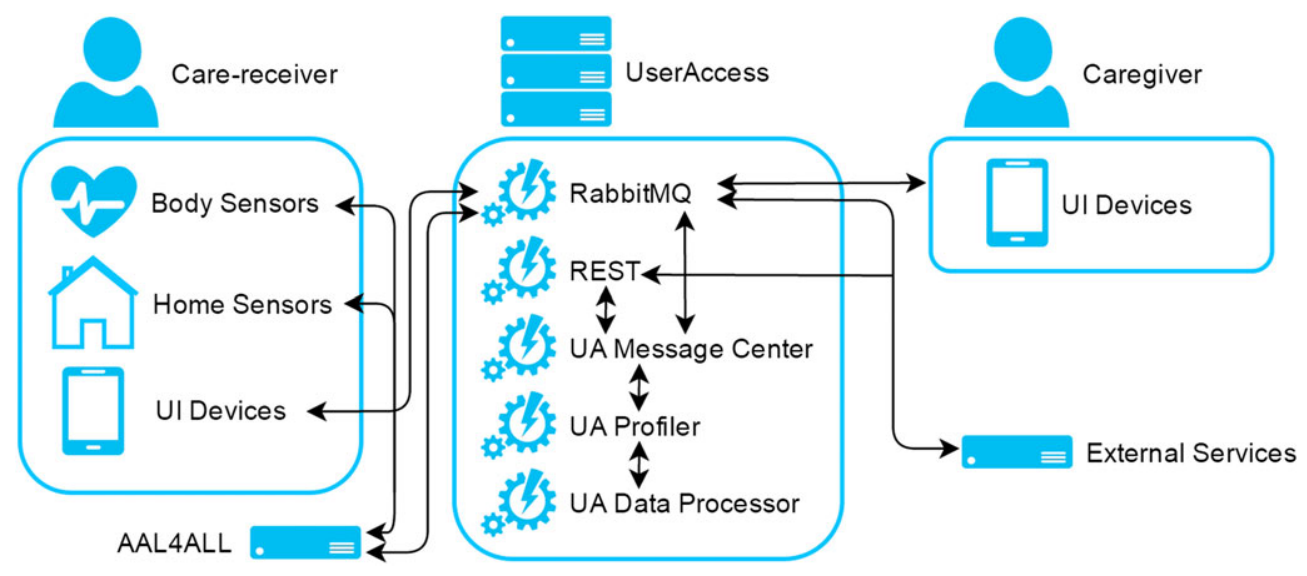

Figure 6: UserAccess structure overview.

2015) and establishes the communication channels through it, being the same scenario applied to the caregiver. The external services can use the RabbitMQ or REST, while REST is unadvised for communication, it is maintained for legacy purposes, like the connection with the iGenda.

The REST service is the frontend to an agent running in the MAS. This agent consumes the requests and sorts them to the agent in charge of the user the request is directed to. The REST interface is developed using the SPARK framework that is scalable and is able to process several requests at the same time. Furthermore, there are several REST endpoints in each load-balancing server.

The internal data processing of the UA consists of three modules that internally are agent systems; this facilitates the maintenance and the communication process. In more detail, these modules are the following:

- Message centre: it controls the in and out communication flow of the application, working directly under the communication structures. For each sensor system and user, an agent is required to descramble the communication and adjust it for internal consumption. It also translates the message to be intelligible to be presented on UI devices. Unlike the AM from the iGenda, the message centre does not sort the messages for the correct agents, which is performed by the communication structures.

- Profiler: it helps the message centre to adjust the received content to meet the users' requirements. For each user, the definitions are adjusted initially through the administration webpage. Thereon, the preferences are learned from the user interactions with the mobile client, and the profile is updated according the dismissal of notifications. For instance, if a warning about an open window is repeatedly dismissed without action, then the rule for this warning is demoted, and its priority is diminished. This process is performed offline. Moreover, the profiler keeps the configurations to each user and its realm of connections for fast access in case of critical situation. 
- Data processor: it processes the parsed information the message centre sends and builds datasets of information. This information stands as history of events and transactions, with the additional feature of translating data into human-readable sentences. Moreover, it serves as an interoperable database for external services and a message buffer to unconnected devices.

The UA was expected to become one of the main tool for services developed in the AAL4ALL and in other platforms because of the interoperability features. The adaptability and attention to the users make the UA an indispensable tool to the caregivers.

4.1.1. UserAccess and iGenda At this point, it is clear that there are many similarities and some differences between the UA and the iGenda. Although the area of operation is the same, the goals and architecture are different at their core. A comparison is presented in Table 1 to highlight the features of the two projects.

\subsection{Tests}

The number of tests performed to the platform was limited because of the ending of the AAL4ALL project; thus, testing with real users was not possible. Despite of that, the tests performed internally demonstrated very positive results. To test the UA, a sensor platform developed at the University of Minho specifically designed for the AAL4ALL project was used. The sensor platform consisted of three parts: the sensors, the base receiver and the web service. The sensors, depicted in Figure 7, are the following:

- Luminance: it detects the amount of light present, with the precision needed to distinguish artificial light and sun.

- Motion: it is a basic sensor only activated when a fairly amount of movement is performed.

- Door: it is a magnetic sensor that changed status when the door was open/closed.

- Humidity and temperature: it detects the amount of humidity and overall temperature on a home environment.

- Gas and water: it detects if there is flow of gas or water on the pipes, that is, they have to be installed in the plumbing system.

- Button: it is pressed if there is a panic situation for the user.

- Electric plug: it is a sensor/actuator able to detect if there is current being consumed and flip the on/off status by remote command.

The sensor system used ZigBee protocol for communication with the base receiver, which was favoured over Bluetooth for its flexibility, number of nodes and energetic efficiency. The base receiver then transported the raw data through a parser, and the outcome was a higher level JSON-formatted message, following the specifications of the AAL4ALL communication protocols. Furthermore, the web service also aimed to a low throughput of data as the AAL4ALL receiving nodes had already a large amount of data passing through; thus, the data sent were the mean

Table 1: Comparison of the UserAccess and iGenda features

\begin{tabular}{lll}
\hline Area & & \multicolumn{1}{c}{ iGenda } \\
\hline Main users & Care receivers & Caregivers \\
Architecture & Multi-agent system & Multi-agent system \\
Communication & JADE FIPA & JADE FIPA and REST \\
Interfaces & Directed to care receivers & Directed to caregivers \\
Platform & Android and web & Android and web \\
Performance & 10s average (from received event to scheduled) & 3 s average (from sensor trigger to notification) \\
Objectives & Intelligent scheduler & AAL4ALL caregiver interface \\
\hline
\end{tabular}

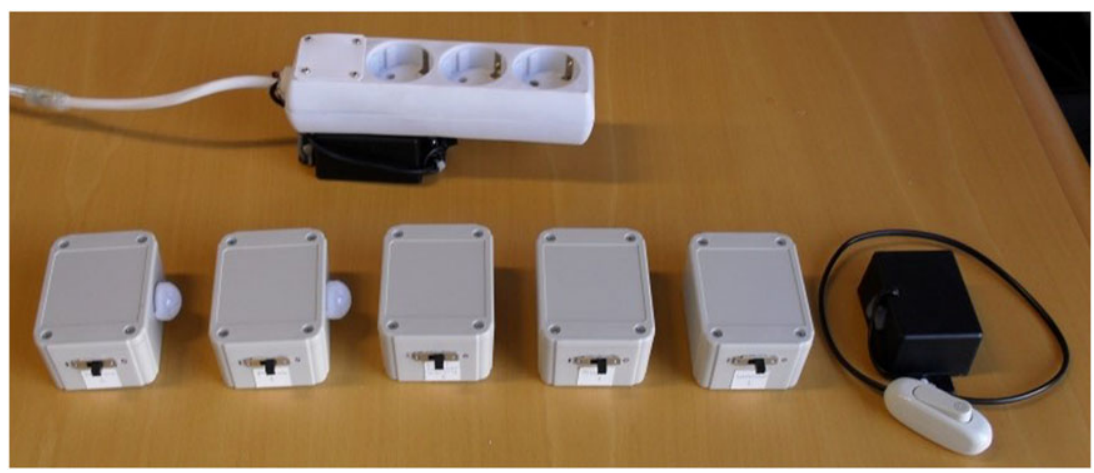

Figure 7: Some of the sensors used in testing (pre-production version). 
of the last $5 \mathrm{~s}$ of data collected, excepting the case of the button press, which was relayed immediately.

This sensor platform provided the information displayed in Figures 4 and 5 , thus proving the feasibility of the UA. The communication was successful, as well as the information parsing and display. The version of the UA tested was the one directed to the formal caregivers, seen in Figures 4 and 5.

The 'panic button' tested positive, and the message was presented in the screen, having 2-5s of time discrepancy, which we consider excellent as initial results. Additionally, the electric plug was successfully controlled in the role of disconnecting the light if it was forgotten that is was turned on. In this case, due to the sensor limitations, we could not detect if the light is currently turned on or off when using efficient bulbs, for example, LED, due to the lack of sensitivity of the sensor.

We have experimented in a controlled environment recurring to students to interpret the role of users. They interacted with the sensors that were placed in a laboratory. The placement was targeted to emulate a living room. Furthermore, an array of 25 agents simulating other users (in this case, directly simulating the generation of sensor values) was deployed to simulate a nursing home environment. The real sensor platform buffers the data and sends it in 7-s blocks. The emulated sensors followed this time requirement, and the actions they report are according a set of actions registered in previous tests. This decision was made to filter random actions that would not happen in real-life scenarios. The test environment was set up in the following way: the communications services were present at a dedicated machine, the UA platform was present in five machines (for load balancing) each with 7 assigned users and one with 4 users, and the databases were on a dedicated machine. Table 2 shows the response time values registered upon the execution. The mean response value corresponds to the time period between receiving the sensor platform data and the visual representation of a warning. The errors represent the number of tests that failed to be registered or shown on the interface. The loads correspond to the number of users active at the same time, from 1 (light) to 26 (heavy). The action was the following sequence: open the door, move inside, open water and gas, turn on a lamp exit in the room and press the panic button. Each sequence was performed four times by each user.

\section{Future path and conclusions}

The natural evolution to the iGenda and the UserAcess is the Cognitive Life Assistant (CLA) platform. The CLA joins the strongest features of both referred projects and includes

Table 2: UserAccess mean response values

\begin{tabular}{lcc}
\hline Load & Mean response value & Errors \\
\hline Light load & $<1 \mathrm{~s}$ & 0 \\
Medium load & $1.7 \mathrm{~s}$ & 0 \\
Medium-high load & $3 \mathrm{~s}$ & 2 \\
Heavy load & $8 \mathrm{~s}$ & 22 \\
\hline
\end{tabular}

intelligent social computing (Sheth et al., 2013; Wang et al., 2007) and the human behaviour emulation (Gomes et al., 2014; Pimenta et al., 2015; Carneiro et al., 2013).

As the name suggests, the CLA is directed to people with cognitive limitations, and one of its goals is to make decisions on daily events in the best interest of the user. Another goal is to improve the way that profiles are constructed and introducing real-time processing in the profiler. Furthermore, 'influence' will be introduced; each user action will influence the users on his or her social circle by changing their preferences.

The CLA will be established on three actions: behaviour analysis, intelligent scheduling and emotion analysis. The architecture will be similar to MAS but with a lighter footprint, because previous developments could scale correctly but suffered from a large consumption of hardware. Moreover, some actions will be implemented directly on mobile devices, as they currently possess the hardware needed for communication processing.

Although the iGenda, UA and CLA do share the same line of investigation, their objective is very distinct from each other. However, their main advantage is their interoperability, which facilitates feature sharing.

In our view, the main structural advance that the newest AAL projects can present is its interoperability. Interoperability ensures that all projects are usable and can benefit from other projects by interacting with them. For instance, a home environment could be equipped with different products and/or services, and one integrated solution would display and reason the data received or use other processes to communicate with the user.

Moreover, in the case of the AAL solutions, it is imperative that the solutions are adapted to its users. Because most of the users are debilitated in some way, a universal solution is inappropriate as it could lack the flexibility or features needed to perform certain tasks. Personalization is a key factor to AAL platforms. The user's environment must be envisioned to provide functionalities adjusted to their recipient. Also, the personalization must reach the services' core adjusting the response in order to cope with certain actions that do not follow the general procedures. This is especially important to people with cognitive disabilities that may lack decision abilities.

This paper presents one solution related to the area of the AAL, and the work developed by the Intelligent Systems Lab at the University of Minho. The scientific acceptance, prototypes and final applications and field tests have proven feasibility of this work. Furthermore, current-related projects follow closely some of the features implemented on the iGenda and the UA, thus using them as a steppingstone to developments either directly related to the work presented or to other research areas.

\section{Acknowledgements}

This work has been supported by FCT - Fundação para a Ciência eTecnologia within the Project Scope: UID/CEC/ 
00319/2013 and COMPETE: POCI-01-0145-FEDER007043. A. Costa thanks the Fundação para a Ciência e a Tecnologia (FCT) the post-doc scholarship with the ref. SFRH/BPD/102696/2014. This work is also partially supported by the MINECO/FEDER TIN2015-65515-C4$1-\mathrm{R}$.

\section{References}

AAL4ALL, 2015. AAL4ALL - ambient assisted living for all. Available at: www.aal4all.org.

Aarts, E. and J.L. Encarnação (Eds) (2006) True Visions: The Emergence of Ambient Intelligence, Berlin: Springer.

Aarts, E., R. Harwig and M. Schuurmans (2001) Ambient intelligence. In The Invisible Future, New York: McGraw-Hill, $235-250$.

Adena, M. and M. Myck (2014) Poverty and transitions in health in later life, Social Science and Medicine, 116, 202-210.

Atzori, L., A. IERA and G. Morabito (2010) The Internet of things: a survey, Computer Networks, 54, 2787-2805.

Bellifemine, F.L., G. CAire and D. Greenwood (2007) Developing Multi-agent Systems with JADE, New York: Wiley.

Boffetta, P., et al. (2014) The Consortium on Health and Ageing: Network of Cohorts in Europe and the United States (CHANCES) project - design, population and data harmonization of a large-scale, international study, European Journal of Epidemiology, 29, 929-936.

Camarinha-Matos, L.M. and H. Afsarmanesh (2004) A multiagent based infrastructure to support virtual communities in elderly care, Int $J$ of Networking and Virtual Organisations, 2(3), 246-266.

Care4Balance, 2015. http://www.aal-care4balance.eu/.

CARneiro, D. et al., 2013. Enriching conflict resolution environments with the provision of context information. Expert Systems.

Cook, D.J., J.C. Augusto and V.R. Jakkula (2009) Ambient intelligence: technologies, applications, and opportunities, Pervasive and Mobile Computing, 5(4), 277-298.

CostA, Â., et al. (2011) Increased performance and better patient attendance in an hospital with the use of smart agendas, Logic Journal of IGPL, 20(4), 689-698.

CostA, Â., et al. (2010a) Multi-agent personal memory assistant. In Demazeau, Y., et al. (editors), Trends in Practical Applications of Agents and Multiagent Systems. Advances in Intelligent and Soft Computing, Berlin, Springer Berlin Heidelberg, 97-104.

Costa, Â. and P. NovaIs (2011) An intelligent multi-agent memory assistant. In Bos, L., et al. (editors), Handbook of Digital Homecare - Successes and Failures. Communications in Medical and Care Compunetics, Berlin: Springer, 197-221.

Costa, A., P. Novais and R. Simoes (2014) A caregiver support platform within the scope of an ambient assisted living ecosystem, Sensors, 14(3), 5654-5676.

CostA, R., et al. (2010b) User recognition in AAL environments. In Augusto, J.C., et al. (editors), Ambient Intelligence and Future Trends - International Symposium on Ambient Intelligence (ISAmI 2010). Advances in Soft Computing, Springer Berlin Heidelberg, Berlin, Heidelberg, 177-184.

Department of Economic and Social Affairs, P.D, 2013. World Population Ageing 2013,

FUXREITER, T. et al., 2010. A modular platform for event recognition in smart homes. In 12th IEEE International Conference on e-Health Networking, Application and Services, Healthcom 2010 Lecce, Italy.

FuXreITER, T., et al. (2011) Rule-based indoor localization using non-intrusive domotic sensors and the HOMER platform. In
Partnerships for Social Innovation in Europe. Proceedings of AAL Forum 2011, 569-576.

Gawinecki, M. and G. Frackowiak (2008) Multi-agent systems with JADE: a guide with extensive study, IEEE Distributed Systems Online, 9(3), $4-4$.

Gershenfeld, N., R. Krikorian and D. Cohen (2004) The Internet of things, Scientific American, 291, 76-81.

Gomes, M., et al. (2014) Studying the effects of stress on negotiation behavior, Cybernetics and Systems, 45, 279-291.

HOMER, 2015. http://homer.aaloa.org/. Available at: http:// homer.aaloa.org/

Kranz, M., P. Holleis and A. Schmidt (2010) Embedded interaction: interacting with the Internet of things, IEEE Internet Computing, 14, 46-53.

Mair, C.A., Quinones, A.R. \& Pasha, M.A., 2015. Care preferences among middle-aged and older adults with chronic disease in Europe: individual health care needs and national health care infrastructure. The Gerontologist.

Pimenta, A., et al. (2015) Detection of distraction and fatigue in groups through the analysis of interaction patterns with computers. In Intelligent Distributed Computing VIII. Studies in Computational Intelligence, Cham, Switzerland: 29-39.

RabbitMQ, 2015. http://www.rabbitmq.com/.

Ramos, C., J.C. Augusto and D. Shapiro (2008) Ambient intelligence - the next step for artificial intelligence, IEEE Intelligent Systems, 23(2), 15-18.

RAmOs, J., et al. (2014) Interactive guiding and localization platform, International Journal of Artificial Intelligence, 12(1), 63-78.

RAmos, J., et al. (2012) Orientation system for people with cognitive disabilities. In Novais, P., et al. (editors), Ambient Intelligence Software and Applications. Advances in Intelligent and Soft Computing, Roorkee, Springer Berlin, Heidelberg, 43-50.

RelaxedCare, 2015. http://www.relaxedcare.eu/en/.

Sheth, A., P. Anantharam and C. Henson (2013) Physical-cybersocial computing: an early 21 st century approach, IEEE Intelligent Systems, 28, 78-82.

UnderstAid, 2015. http://understaid.com/.

United Nations, 2009. World population ageing, Available at: http://www.un.org/esa/population/publications/WPA2007/ SummaryTables_new.pdf.

United Nations, Department of Economic and Social Affairs, P.D, 2015. Population ageing and development database 2014.

VastenburG, M.H., et al. (2008) Ambient intelligence. In Aarts, E., et al. (editors), European Conference on Ambient Intelligence, AmI '08. Lecture Notes in Computer Science, Springer Berlin Heidelberg, Berlin, Heidelberg, 1-12.

WANG, F., et al. (2007) Social computing : from social informatics, IEEE Intelligent Systems, 22, 79-83.

Wooldridge, M. (1997) Agent-based software engineering. In Bradshaw, J. and G. Arnold (editors), IEE Proceedings Software Engineering 144, 26.

\section{The authors}

\section{Angelo Costa}

Ângelo Costa has received his European Doctorate title at the University of Minho (Portugal) in 2013 on the biomedical area. He has also received his Master's title in 2009 at the University of Minho and is a lecturer at the Polytechnic of Porto. He was the author and co-author of an extensive number of publications, in international journals with impact factor, international book chapters, and in conference proceedings with international peer review. He is currently in a post-doc developing the Cognitive Life Assistant, a partnership of the University of 
Minho and the Universitat Politècnica de València, and one of the main developers of cognitive assistants for mobile platforms.

\section{Vicente Julián}

Vicent J. Julian Inglada received his $\mathrm{PhD}$ from the Universitat Politècnica de València (UPV) in 2002. He holds a position of associate professor of Computer Science at the Universitat Politècnica de València. He is a member of the 'Grupo de Tecnología Informática-Inteligencia Artificial', and deputy director of the Official Master in Artificial Intelligence, Pattern Recognition and Digital Imaging at the UPV. He has more than 40 works published in journals with outstanding positions, papers published in conference proceedings that have a system of external peer review and dissemination of knowledge comparable with journals indexed in relevant positions.

\section{Paulo Novais}

Paulo Novais is an associate professor with Habilitation of Computer Science at the Department of Informatics of the University of Minho (Portugal) and a researcher at the ALGORITMI Centre in which he is the coordinator of the research group Intelligent Systems Lab. From the same university, he received a $\mathrm{PhD}$ in Computer Science in 2003. He has led and participated in several research projects sponsored by Portuguese and European public and private institutions. $\mathrm{He}$ is the co-author of over 170 book chapters, journal papers, conference, and workshop papers and books. 